\title{
Combination Forecasting Model Using Grey Verhulst Models Coupling to Regression Analysis
}

\author{
Qing Shu and Xinping Xiao* \\ College of Science, Wuhan University of Technology, Wuhan 430070, China \\ *Corresponding author
}

\begin{abstract}
Firstly, grey verhulst model based on fractional order accumulate is deduced. Then, take advantage of differential and difference to find the corresponding relationship between the grey verhulst model of each order and the regression equation. Giving the level of significance $a$, carry out the significance test for the regression equations. Picking up the orders of grey verhulst models corresponding to the first two regression equations which are best-fit for the original time series, then establish the grey verhulst models which have the orders picked up before. Lastly, using these two grey verhulst models to establish the weight arithmetic average combination forecasting model based on grey associate analysis. The practical example indicates that the fitting precision of our model is improved significantly comparing with single forecasting models, logistic model and fractional order accumulate grey verhulst model. It is proved to be an available and practical method for forecasting.
\end{abstract}

Keywords- grey verhulst model; fractional order accumulation; significance level; combination forecasting; regression analysis

\section{INTRODUCTION}

Fractional order contains a thought of 'in between'. In these years, it gets a wide range of recognition. More and more scholars use the thought of fractional order accumulation into grey model. The fractional order accumulate discrete grey model has defined in order to reduce perturbation bound and the question why grey model is suitable for small sample has been explained ${ }^{[1]}$. Wu Li-feng ${ }^{[2]}$ has studied GM $(1,1)$ model based on fractional order accumulating method and its stability. He has expanded integer order accumulating to fractional order accumulating. Fractional order accumulating has reduced the perturbation bound and relieved the contradictory between the less impact on solution by the new information and the principle of new information priority. Mao Shu-hua ${ }^{[3]}$ has transformed traditional time-lag $\operatorname{GM}(1, \mathrm{~N}, \tau)$ model into fractional order accumulation time-lag $\operatorname{GM}(1, N, \tau)$ after introducing fractional order accumulation generation operator. The example indicated that the accuracy of fractional order accumulation time-lag $\mathrm{GM}(1, \mathrm{~N}, \tau)$ is more satisfactory. Yang Bao-hua ${ }^{[4]}$ has transformed grey discrete power GM $(1,1)$ into fractional order grey discrete power $\operatorname{GM}(1,1)$, and application examples showed the higher precision accuracy of the new model. Chen $\mathrm{Ye}^{[5]}$ has extended the original $\mathrm{r}$-AGO technique from integer domain to the real number field, and put forward the corresponding GM $(1,1)$ model with its solving process.

When evaluate a model, fitting and forecasting effect and the stability of the model are used ${ }^{[1]}$. Fractional order accumulate grey model remits the shortage on the stability of traditional grey model, and reduces the perturbation bound. Application examples also show that compared to the traditional grey model, fitting accuracy of fractional order accumulate grey model is improved to some extent. In this paper, fractional order accumulate grey verhulst model is deduced at first. Then, a new combinational forecasting method based on that model is proposed. Comparing to the fractional order accumulate grey verhulst model, this combination forecasting model can improve the fitting accuracy significantly.

\section{COMBINATION ForeCAST MODEL USING GREY} Verhulst MODELS COUPLING TO REGRESSION ANALYSIS

A. Fractional Order Accumulate Grey Verhuslt Model Definition 2.1. Suppose $y^{(0)}=\left(y^{(0)}(1), y^{(0)}(2), \mathrm{L}, y^{(0)}(n)\right)^{T}$ is original sequence, $d$ is transform operator of original sequence, $x^{(0)}=\left(x^{(0)}(1), x^{(0)}(2), \mathrm{L}, x^{(0)}(n)\right)^{T}$ is transform sequence. $y^{(0)}(k) d=x^{(0)}(k), k=1,2, \mathrm{~L}, n ; A^{r}$ is accumulative generator matrix ${ }^{[5]}$ when the order is $r$. Then, the sequence $x^{(r)}$ is called r-order accumulative generator sequence which is generated from $x^{(r)}=A^{r} x^{(0)}$.

$$
\begin{aligned}
& x^{(r)}= {\left[\begin{array}{c}
x^{(r)}(1) \\
x^{(r)}(2) \\
\mathrm{M} \\
x^{(r)}(n)
\end{array}\right]=\left[\begin{array}{cccc}
1 & 0 & \mathrm{~L} & 0 \\
r & 1 & \mathrm{~L} & 0 \\
C_{r+1}^{2} & r & \mathrm{~L} & 0 \\
\mathrm{M} & \mathrm{M} & \mathrm{O} & \mathrm{M} \\
C_{r+n-2}^{n-1} & C_{r+n-3}^{n-2} & \mathrm{~L} & 1
\end{array}\right] \mathrm{g} } \\
& {\left[\begin{array}{c}
x^{(0)}(1) \\
x^{(0)}(2) \\
\mathrm{M} \\
x^{(0)}(n)
\end{array}\right]=A^{r} x^{(0)} }
\end{aligned}
$$

Definition 2.2. Grey verhulst model based on fractional order accumulate: 


$$
x^{(r)}(k)-x^{(r)}(k-1)+a z^{(r)}(k)=b\left(z^{(r)}(k)\right)^{2}
$$

There is $x^{(r)}=A^{r} x^{(0)}, z^{(r)}(k)=0.5 x^{(r)}(k)+0.5 x^{(r)}(k-1)$.

Theory 2.1. (1) The lever 1 parameters pack of fractional order accumulate grey verhulst model is $P=(a, b)^{T}$, there is matrix formula under the least squares principle:

$$
P=\left[\begin{array}{l}
a \\
b
\end{array}\right]=\left(B^{T} B\right)^{-1} B^{T} X
$$

And

$$
B=\left[\begin{array}{cc}
-z^{(r)}(2) & \left(z^{(r)}(2)\right)^{2} \\
-z^{(r)}(3) & \left(z^{(r)}(3)\right)^{2} \\
\mathrm{M} & \mathrm{M} \\
-z^{(r)}(n) & \left(z^{(r)}(n)\right)^{2}
\end{array}\right], X=\left[\begin{array}{c}
x^{(r)}(2)-x^{(r)}(1) \\
x^{(r)}(3)-x^{(r)}(2) \\
\mathrm{M} \\
x^{(r)}(n)-x^{(r)}(n-1)
\end{array}\right] .
$$

(2) The level 2 parameters pack of fractional order accumulate grey verhulst model is $(s(i), D, E)$ with $s(i)=\sum_{k=2}^{n}\left(z^{(r)}(k)\right)^{(i)}, D=\sum_{k=2}^{n} x^{(r-1)}(k) z^{(r)}(k), E=\sum_{k=2}^{n} x^{(r-1)}(k)\left(z^{(r)}(k)\right)^{2}$ so

$$
P=\left[\begin{array}{l}
a \\
b
\end{array}\right]=\frac{1}{s(2) s(2)-s^{2}(3)}\left[\begin{array}{l}
s(3) E-s(4) D \\
s(2) E-s(3) D
\end{array}\right]
$$

Theory 2.2. Under the boundary condition $\hat{x}^{(r)}(1)=x^{(r)}(1)$, the white response of fractional order accumulate grey verhulst model is

$$
\hat{x}^{(r)}(k+1)=\frac{a x^{(r)}(1)}{b x^{(r)}(1)+\left(a-b x^{(r)}(1)\right) e^{a k}}
$$

With

$$
\hat{x}^{(0)}=\left(A^{r}\right)^{-1} \hat{x}^{(r)}, \hat{y}^{(0)}=\hat{x}^{(0)} d^{-1}
$$

Intelligent algorithms such as genetic algorithm are used to search order $r$.

\section{B. Choose the Optimal Grey Verhuslt Model Based on Regression Equation}

Grey Verhulst model usually uses 1-AGO on the original sequence ${ }^{x^{(0)}(t)}$ to generate accumulative sequence ${ }^{x^{(1)}(t)}$, and then we can get the mean value generating sequence ${ }^{z^{(1)}}(t)$.
Lastly, $x^{(0)}(t), x^{(1)}(t)$ and $z^{(1)}(t)$ are used to set up grey verhulst model:

$$
x^{(0)}(k)+a z^{(1)}(k)=b\left(z^{(1)}(k)\right)^{2}
$$

And its whitenization equation is

$$
\frac{d x^{(1)}(t)}{d t}+a x^{(1)}(t)=b\left(x^{(1)}(t)\right)^{2}
$$

Define ${ }^{x^{(1)}(t)=\int_{0}^{t} x^{(0)}(s) d s \approx \sum x^{(0)}(t) \Delta t}$. When $\Delta t$ is small, the derivative of $x^{(1)}(t)$ to the time $t$ is $x^{(1)^{\prime}}(t)=x^{(0)}(t) \approx \frac{x^{(1)}(t+\Delta t)-x^{(1)}(t)}{\Delta t}$.

. Especially take $\Delta t=1$, there is $x^{(1)^{\prime}}(t) \approx x^{(1)}(t+1)-x^{(1)}(t)$, so $x^{(1)}(t) \approx \sum x^{(0)}(x)$, $x^{(1)^{\prime}}(t)=x^{(0)}(t) \approx x^{(1)}(t+1)-x^{(1)}(t)$, then the formula (7) can be turned to:

$$
x^{(1)}(k)-x^{(1)}(k-1)+a x^{(1)}(k)=b\left(x^{(1)}(k)\right)^{2}
$$

And

$$
\frac{x^{(1)}(k)-x^{(1)}(k-1)}{x^{(1)}(k)}+a=b\left(x^{(1)}(k)\right)
$$

It equivalent to

$$
\frac{x^{(0)}(k)}{x^{(1)}(k)}=b x^{(1)}(k)-a
$$

Set $\frac{x^{(0)}(k)}{x^{(1)}(k)}=Y(k)$ as dependent variable and $x^{(1)}(k)$ as independent variable. The linear relationship between $Y(k) \quad$ and $\quad x^{(1)}(k)$ is represented as $Y(k)=b x^{(1)}(k)-a$. If the regression equation could fit the original sequence $x^{(0)}=\left(x^{(0)}(1), x^{(0)}(2), \mathrm{L}, x^{(0)}(n)\right)^{T}$ well, then the corresponding grey verhulst model (6) has a better fitting effect.

Significance test is given to the linear regression equation $Y(k)=b x^{(1)}(k)-a$ while significance level is $a$. If 
the test is passed, the traditional grey verhulst model $x^{(1)}(k)-x^{(1)}(k-1)+a z^{(1)}(k)=b\left(z^{(1)}(k)\right)^{2}$ can be set up.

$$
\text { Similarly, if } Y(k)=\frac{x^{(1)}(k)}{x^{(2)}(k)} \text { is dependent variable }
$$
and ${ }^{x^{(2)}(k)}$ is independent variable, the grey verhulst model generated from 2-AGO: $x^{2}(k)-x^{(2)}(k-1)+a z^{(2)}(k)=b\left(z^{(2)}(k)\right)^{2}$ can be set up as the same way.

The order of the accumulative sequence within the independent corresponds to the order of grey verhulst model. Giving the significance lever $a$ and repeating these significance tests until the regression equation can't pass through.

The value of coefficient of determination $R^{2}$ reflects to the fitting degree of regression equation. Comparing all $R^{2}$ of the regression equations which are pass the significance test and choosing the best two regression equations, the corresponding orders of the accumulative sequence within the independent are orders of the most optimal single grey verhulst models.

\section{Combination Forecasting Model Based on Grey Correlation Analysis}

Choose the best two optimal single grey verhulst models to set up weighted arithmetic means combination forecasting model based on grey correlation analysis.

Wang Yingming ${ }^{[6]}$ put forward that forecasting results can be evaluated comprehensively from five aspects at least. They are Sum Squared Error (SSE), Mean Absolute Error (MAE), Mean Squared Error (MSE), Mean Absolute Percentage Error (MAPE) and Mean Squared Percentage Error (MSPE). If just choose one of these error indicators to evaluate the forecasting result, it is not that comprehensive. In addition to these five cost indices, this paper intend to add a benefit index-grey absolute correlation degree - to evaluate the forecasting result of the combination model. Tang Shaoliang ${ }^{[7]}$ gives the definition of grey absolute correlation degree between single forecast sequence, combination forecast sequence with original sequence.

Take the method of reference [8], standardize the decision matrix and get the weight vector of indicators, then the positive and negative ideal solutions can be confirmed. Calculate the optimum subordinate degrees and normalize them, and then we can get the weight of solutions.

\section{EXAMPLE ANALYSIS}

Choose data of carbon emissions ${ }^{[9]}$ (Table 1) of Shanxi province from 2002 to 2010 to set up combination forecasting model this paper proposed upon.

\section{A. Choose the Optimal Grey Verhuslt Model Based on Regression Equation}

Take $X^{(0)}$ as the original sequence, suppose dependent variable is $Y(t)=\frac{X^{(-1)}(t)}{X^{(0)}(t)}$, and independent variable is $X^{(0)}(t)$, then we can get $a=0.9185, b=-0.0001$ and the regression equation $y=-0.0001 x+0.9185$. Giving significance level $\alpha=0.05$, we can get $R^{2}=0.6299$, obviously pass through the significance test. Then we can set up straight grey verhulst model ${ }^{[10]}$ from the regression equation upon.

Similarly, suppose dependent variable is $Y(t)=\frac{X^{(0)}(t)}{X^{(1)}(t)}$, then we can get the regression equation $y=-0.00002 x+0.6795$. Giving significance level $\alpha=0.05$, we can get $R^{2}=0.6126$, obviously pass through the significance test. This regression equation corresponds to traditional grey verhulst model.

Set up corresponding regression equation successively. When the significance level is $\alpha=0.05$, give significance test to all these regression equation. Then transfer all the regression equation which pass through the significance test into grey verhulst model which has the corresponding order, such as 2-AGO grey verhulst model, 3-AGO gery verhulst model and so on.

In all the regression equations which have passed through significance test, the largest two $R^{2}$ which show best fitness to original sequence are correspond to straight grey verhulst model and traditional grey verhulst model. Set up corresponding single grey verhulst model $\mathrm{M}_{1}$ and $\mathrm{M}_{2}$ separately.

As to formula (2), substitute into $r=0$, straight grey verhulst model can be get according to theory 2.1.1: $x^{(0)}(k)-x^{(0)}(k-1)-0.2374 z^{(0)}(k)=-0.00003397\left(z^{(0)}(k)\right)^{2}$.Its solution is $\hat{x}^{(0)}(k+1)=\frac{473.661}{0.0678+0.1696 e^{-0.2374 k}}, k=0,1, \ldots$. We can get the fitting data of carbon emission in Shanxi province and all indicators we will take to evaluate the model. The results will be shown in Table 1 . And substitute $r=1$ to formula (2) in the same way.

\section{B. Combination Forecasting Model Based on Grey Correlation Analysis}

Choose the best two optimal single grey verhulst models upon to set up weighted arithmetic means combination forecasting model based on grey correlation analysis. The standardized decision matrix can be gotten. And the weight of indicators

is $\omega=[0.235552,0.183359,0.185701,0.186147,0.189086,0.020154]$. Then, we can get the positive and negative ideal solutions

Calculate the grey correlation degree and subordinate degree between solutions $\mathrm{M}_{\mathrm{i}}$ with $\mathrm{M}^{+}$and $\mathrm{M}^{-}$. Then we can 
get the weight of solutions $k_{1}=0.871367, k_{2}=0.128633$, and the results of combination forecasting model are shown in Table I.

\section{Comparing Fitting Effect Between Different Models}

The fractional order grey verhulst model is set up using genetic algorithm which base on the object that minimum MAPE to search the optimal order which is $r=0.3740$, and parameters are $a=-0.3590, b=-0.00003$. The fitting values and corresponding indicators are also shown in Table I.

TABLE I. FITTING VALUES AND VALUE OF EVALUATE INDICATORS

\begin{tabular}{|c|c|c|c|c|c|c|}
\hline Year & $\begin{array}{c}\text { Data of Carbon } \\
\text { Emission }\end{array}$ & $\mathrm{M}_{1}$ & $\mathrm{M}_{2}$ & $\begin{array}{c}\text { Combination } \\
\text { Forecast Model }\end{array}$ & Logistic Model & $\begin{array}{l}\text { Fractional Order } \\
\text { Grey Verhuslt Model }\end{array}$ \\
\hline 2002 & 1995.5 & 1995.5 & 1995.5 & 1995.5 & 2233.8 & 1995.5 \\
\hline 2003 & 2446.6 & 2350.283 & 965.9958 & 2185.95 & 2494.6 & 1942.993 \\
\hline 2004 & 2950.8 & 2733.602 & 1388.821 & 2573.959 & 2786 & 2312.352 \\
\hline 2005 & 3366.7 & 3137.144 & 1947.822 & 2995.956 & 3111.4 & 2807.031 \\
\hline 2006 & 3715.2 & 3550.535 & 2638.909 & 3442.313 & 3474.7 & 3350.943 \\
\hline 2007 & 3970 & 3962.341 & 3412.418 & 3897.058 & 3880.6 & 3881.852 \\
\hline 2008 & 4373.5 & 4361.3 & 4156.285 & 4336.962 & 4333.8 & 4342.443 \\
\hline 2009 & 4580.6 & 4737.52 & 4708.558 & 4734.081 & 4839.9 & 4690.671 \\
\hline 2010 & 5152.5 & 5083.372 & 4915.821 & 5063.482 & 5405.2 & 4908.514 \\
\hline SSE & & 460002.082 & 8234054.384 & 165871.833 & 349930.5 & 1187528 \\
\hline MAE & & 181.455692 & 741.909567 & 105.960077 & 176.444444 & 282.1381 \\
\hline MSE & & 678.234533 & 2869.504205 & 407.273659 & 591.549237 & 1089.737 \\
\hline MAPE & & 0.055036 & 0.234404 & 0.030875 & 0.052522 & 0.087464 \\
\hline MSPE & & 0.216817 & 0.965947 & 0.122255 & 0.184133 & 0.360269 \\
\hline Absolute of Grey Incidence & & 0.934421 & 0.833432 & 0.950512 & 0.940991 & 0.905239 \\
\hline
\end{tabular}
[9].

The fitting values of logistic model come out of reference

From Table 1, it shows that comparing to single grey verhulst models, fractional order accumulate grey verhulst model and logistic model, combination forecasting model using grey verhult models with different orders coupling to regression analysis proposed in this paper has smallest value in five error indicators and largest value in grey absolute correlation degree. It has the largest value of grey absolute correlation degree which is 0.9505 , and has the smallest value of MAPE which is $3.09 \%$. Comparing to MAPE of fractional order grey verhulst model, it reduces nearly 6\%. It indicates that the method proposed in this paper improves the fitting precision, and has a better fitting effect than fractional order accumulate grey verhulst model.

\section{CONCLUSIONS}

Traditional combination forecasting model combines more than two different kinds of models, but in this paper, the same kind of models with different orders are combined. Because bases on the grey model's character of AGO (Accumulated Generating Operation), this method can excavate the information from original sequence fully, and provide new idea for combination forecasting model. This paper proposes using the significance test of regression equation to choose appropriate single grey verhulst models with different orders and combining to forecast. Owing to the corresponding relations between regression equations and grey verhulst models with each order, the regression equation which has the best fitting effect corresponds to the grey verhulst model with best fitting effect. This can be very persuasive. The practical example proves that the method this paper proposes improves the fitting precision and provides a new idea for combination forecasting model.

\section{ACKNOWLEDGMENT}

We are very grateful to the research group of Grey System in Wuhan University of Technology for providing meaningful suggestions while this paper is being written. This paper is supported by National Natural Science Foundation of China (Grant No. 71540027, Grant No. 51479151), and Research 
Fund for the Doctoral Program of Higher Education of China (Grant No. 20120143110001).

\section{REFERENCES}

[1] Wu Li-feng, Liu Si-feng and Yao Li-gen, "Discrete grey model based on fractional order accumulate,” Systems Engineering-Theory \&Practice, Vol.34, No.7, July 2014, pp. 1822-1827.

[2] Wu Li-feng, Liu Si-feng and Liu Jian, "GM(1,1) model based on fractional order accumulating method and its stability," Control and Decision, Vol.29, No.7, May 2014, pp. 919-924.

[3] Mao Shu-hua, Gao Ming-yun, Xiao Xin-ping, "Fractional order accumulation time-lag GM(1,N, $\tau)$ model and its application,” Systems Engineering-Theory \&Practice, Vol.35, No.2, Feb 2015, pp. 430-436.

[4] Yang Bao-hua, Zhao Jin-shuai, "Fractional order discrete grey $\operatorname{GM}(1,1)$ power model and its application," Control and Decision, Vol.30, No.7, Jul 2015, pp. 1264-1268.

[5] Chen Ye, Modeling Research on Fractional Order Accumulation in Short-Term Traffic Flow Forecasting, Wuhan: Wuhan University of Technology, 2013

[6] Wang Ying-ming, "Research on the methods of combining forecasts based on correlativity,” Forecasting, Vol.21, No.2, Feb 2002, pp. 58-62.

[7] Tang Shao-liang, Li Nan, Gong Zai-wu, "Research on properties of combination forecasting model based on absolute of grey incidence," System Engineering and Electronic, Vol.30, No.1, Jan 2008, pp. 89-92.

[8] Yan Shu-li, Research on Methods of Multiple Attribute DecisionMaking and Integration, Wuhan: Wuhan University of Technology, 2005

[9] Du Qiang, Chen Qiao, Yang Rui, "Forecast caron emissions of provinces in China based on logistic model”, Vol.22, No.22, Feb 2013, pp. 143151.

[10] Feng Zheng-yuan, “Direct grey model,” Acta Mathematicae Applicatae Sinica, Vol.15, No.3, July 1992, pp. 345-354. 